\title{
Physicochemical and cytotoxicity analysis of glycerol monoolein-based nanoparticles
}

\author{
Terence E. Hartnett, ${ }^{\text {a,b }}$ Katharina Ladewig, ${ }^{\text {a }}$ Andrea J. O'Connor, ${ }^{\text {a }}$ Patrick G. Hartley, ${ }^{\text {b }}$ Keith M. \\ McLean ${ }^{\mathrm{b}_{*}}$
}

Lyotropic liquid crystalline dispersions, such as cubosomes, have been proposed as potential drug delivery vehicles. A recently described 'salt induced' method of cubosome production may be suitable for the encapsulation of macromolecular bioactive therapeutics, such as proteins, within the cubic phase.

Here, we develop and characterise glycerol-monoolein (GMO)-based cubosomes using this novel method 10 of cubosome production.

Using the anionic biological lipid 1,2-dipalmitoylphosphatidylserine (DPPS) to prevent GMO forming its natural cubic-phase, we validate that addition of phosphate buffered saline (PBS) can be used to reverse the effects of DPPS. However, this transition is dependent on the type of Pluronic ${ }^{\circledR}$ block copolymer stabiliser used to prevent re-flocculation of the cubosome dispersions. Using small angle x-ray scattering

15 (SAXS) and cryogenic transmission electron microscopy, we show that the 'salt induced' phase transition from small unilamellar vesicles to cubosomes is inhibited when using Pluronic ${ }^{\circledR}$ F127. In contrast, using the larger, more hydrophilic stabiliser Pluronic ${ }^{\circledR} \mathrm{F} 108$, cubosomes can be formed, although further analysis using SAXS suggests these GMO-based cubosomes are less thermally stable than those comprising GMO alone. In addition, we find no significant difference in the in vitro cytotoxicity of 20 cubosome dispersions formed using either of these stabilisers, or between those containing DPPS and those without. The ability to control cubic phase transitions may present an opportunity for the incorporation of therapeutically relevant proteins in these nanoparticles.

\section{Introduction}

The controlled release of therapeutics from drug delivery vehicles 25 aims to maximise the availability of therapeutics at the site of need for prolonged periods of time and, in so doing, minimise treatment costs. One vehicle that has potential for such delivery is the cubosome. ${ }^{1}$

Cubosomes are lyotropic liquid crystalline (LLC) dispersions 30 based on a specialised group of non-ionic amphiphiles that selfassemble with an intrinsic bilayer curvature when hydrated, resulting in the formation of a nanoporous 3-D network of wateramphiphile channels possessing an internal interfacial area of approximately $400 \mathrm{~m}^{2} / \mathrm{g}$ of amphiphile. ${ }^{2}$ The large internal surface 35 area is produced by the self-assembly of the amphiphiles to form two non-intersecting water networks, which provides cubosomes the capacity to encapsulate hydrophilic, hydrophobic and amphiphilic therapeutics. ${ }^{3}$ Depending on the environmental conditions, three common cubic phases may be observed: the 40 diamond (Pn3m), primitive (Im3m) and gyroid (Ia3d) phases. The phase which forms is dependent on the critical packing parameter (CPP) of the amphiphiles in the bilayer, which, in turn, is dependent on environmental conditions such as temperature. ${ }^{4}$

Cubosomes are traditionally formed through the fragmentation 45 and stabilisation of a bulk cubic phase gel using energy intensive methods such as homogenisation and sonication. As cubic phase forming amphiphiles are commonly uncharged and do not provide steric stabilisation, cubosomes require stabilisation to prevent their re-flocculation following dispersion which is most ${ }_{50}$ commonly achieved using the surfactant Pluronic ${ }^{\circledR}$ F127 (F127). ${ }^{5}$

With the recent and forecast increases in the application of macromolecular bioactive therapeutics, there is a need to develop new and less energy-intensive methods of cubosome production as traditional methods are likely to render these macromolecular 55 therapeutics inactive. ${ }^{1}$ Recently, we have described the production of cubosomes using a 'salt-induced' phase transition from small unilamellar vesicles (SUVs) of phytantriol - 1,2 dipalmitoyl phophatidylserine (DPPS) to cubosomes. ${ }^{6}$ This simple method of cubosome production, first described by Muir 60 et al. (2012), ${ }^{7}$ is made possible by charge-shielding of an ionic lipid. The lipid inserts into the phytantriol bilayer to prevent the cubic phase forming. The cubic phase can then be formed through the addition of an electrolyte such as phosphate buffered saline (PBS).

65 Using the cubic phase forming amphiphile phytantriol, this method of cubosome production has been shown to be amenable to the incorporation of both positively and negatively charged lipids. ${ }^{4,6}$ Phytantriol is regularly used commercially in the cosmetics and hair care industry, ${ }^{8}$ however, owing to the high 70 toxicity it elicits in vitro, its suitability as a drug delivery vehicle remains contentious. On the other hand, glycerol monoolein (GMO), the other commonly used cubic phase forming 
Table 1 - Physical properties of Pluronic ${ }^{\circledR}$ F108 and Pluronic ${ }^{\circledR}$ F127

\begin{tabular}{cccccc}
\hline & $\begin{array}{c}\text { Molecular } \\
\text { Weight }\end{array}$ & $\begin{array}{c}\text { Units of } \\
\text { PO }\end{array}$ & $\begin{array}{c}\text { Units of } \\
\text { EO }\end{array}$ & HLB & Schematic \\
\hline $\begin{array}{c}\text { Pluronic } ₫ \\
\text { F108 }\end{array}$ & 14600 & 44 & 141 & $>24$ & \\
$\begin{array}{c}\text { Pluronic } @ \\
\text { F127 }\end{array}$ & 12600 & 56 & 101 & $18-23$ & \\
\hline
\end{tabular}

amphiphile, has a similar phase behaviour to phytantriol, ${ }^{9,10}$ but is less toxic in vitro. ${ }^{11}$

In its bulk form GMO behaves similarly to phytantriol, however, when dispersed and stabilised with F127, GMO 5 undergoes a phase transition from its natural diamond cubic phase $(\mathrm{Pn} 3 \mathrm{~m})$ to the primitive cubic phase $(\mathrm{Im} 3 \mathrm{~m})$. The phase change, prompted by the addition of F127, results in a smaller effective amphiphile CPP, and hence a shift towards lamellar phase behaviour, with a more swollen structure of larger water 10 channels observed. F127, therefore, acts in an analogous manner to the ionic lipids used in the 'salt-induced' cubosome production technique and may resist the lamellar-to-cubic phase transition required to transform SUV's into cubosomes in this method.

Recently, Chong et al. (2011) looked to uncover the 15 mechanisms of stabiliser control on the cubic phase. They investigated the ability of the Pluronic ${ }^{\circledR}$ series to stabilise both GMO and phytantriol-based cubosomes and established that the molecular weight, cloud point and hydrophilic-lipophilic balance (HLB) all play key roles in the ability of Pluronics ${ }^{\circledR}$ to be 20 effective stabilisers of cubosomes. ${ }^{12}$ They concluded that Pluronic ${ }^{\circledR}$ F108 (F108), a larger, more hydrophilic stabiliser than the traditionally used F127 (Table 1), is a more effective stabiliser of both phytantriol and GMO cubosomes. Significantly, when F108 was used to stabilise GMO-based 25 cubosomes, the natural diamond cubic phase was retained.

Here, we extend our previous work on phytantriol-DPPS cubosomes to demonstrate the use of the 'salt-induced' cubosome production technique using GMO. ${ }^{6}$ Initially, by demonstrating that the transition from SUVs-to-cubosomes is possible using ${ }_{30} \mathrm{~F} 108$, we establish that F108 is a more effective stabiliser than F127 for producing GMO-based cubosomes using the 'saltinduced' production technique. The phase transition is confirmed using synchrotron small angle x-ray scattering (SAXS) and cryogenic transmission electron microscopy (cryo-TEM). ${ }_{35}$ Furthermore, we find that the resulting particles undergo irreversible changes when exposed to high temperatures and demonstrate that the incorporation of DPPS does not alter the cytotoxicity of GMO-based dispersions in vitro.

\section{${ }_{40}$ 2. Materials \& Methods}

\subsection{Materials:}

Glycerol monoolein (GMO) was obtained from Nu-Check Prep Inc. (99\%) (Minnesota, USA), 1,2 - Dipalmitoyl-sn-glycero-3phospho-L-serine, sodium salt (DPPS, Na) was obtained from ${ }_{45}$ Corden Pharmaceuticals (Liestal, Switzerland), Pluronic ${ }^{\circledR}$ F127 (100:65:100) (poly(ethylene oxide) - poly(propylene oxide) poly (ethylene oxide), Pluronic $^{\circledR}$ F108 (132:50:132) (poly(ethylene oxide) - poly(propylene oxide) - poly (ethylene oxide) and phosphate buffered saline tablets were purchased from ${ }_{50}$ Sigma Aldrich (MO, USA). The PBS was prepared at either $1.5 \mathrm{x}$ recommended concentration (1.5x PBS) or at regular concentration (1x PBS) as per manufacturers recommendations. All other materials were used as received

\section{${ }_{55}$ 2.2. Preparation of Dispersions:}

GMO-based dispersions were prepared by dissolving GMO, either F127 or F108 Pluronic ${ }^{(1)}$ (8 wt\%), and the appropriate amount of DPPS in chloroform. The chloroform was removed through rotary evaporation ( 2 hours) and the samples were then 60 placed under vacuum in a desiccator overnight to complete chloroform removal.

Prior to dispersion, the bulk mixture was heated to $50{ }^{\circ} \mathrm{C}$ until viscous. Milli-Q (Millipore, MA, USA) water was added to a final GMO concentration of $2.5 \%(\mathrm{w} / \mathrm{v})$, and the mixture was 65 subsequently dispersed for 3 minutes ( 2 seconds on, 3 seconds off) by ultra-sonication using a MisonixXL2000 (Misonix Inc., NY, USA) at $30 \%$ of maximum output. The resulting dispersions were allowed to stabilise at room temperature for at least 48 hours.

70 Before analysis, either Milli-Q (Millipore, MA, USA) water or 1.5x PBS was added to the dispersions at a volume ratio of 2:1 (2 parts Milli-Q/ 1.5x PBS: 1 part DPPS-GMO dispersion).

\subsection{Particles: Diameter and Polydispersity}

The size and polydispersity of the particles were measured at 75 room temperature by dynamic light scattering (DLS) using a Malvern Zeta-sizer 3000 (Malvern Instruments, Malvern, UK). Prior to analysis, samples were diluted 200x with either 1x PBS or $\mathrm{H}_{2} \mathrm{O}(1 \mu \mathrm{l}: 200 \mu \mathrm{l})$ to minimize the interference that occurs in a highly turbid solution.

80

\subsection{Cryogenic transmission electron microscopy (Cryo-TEM)}

Prior to analysis, samples that required temperature control were placed in a Thermomixer Comfort (Eppendorf, Hamburg, Germany) for one hour at the given temperature.

85 A laboratory-built humidity-controlled vitrification system was used to prepare the samples for cryo-TEM. Humidity was kept close to $80 \%$ for all experiments, and ambient temperature at $22{ }^{\circ} \mathrm{C}$. 200-mesh copper grids coated with perforated carbon film (Lacey carbon film: ProSciTech) were glow discharged in 90 nitrogen prior to application of the sample. Aliquots of the sample $(4 \mu \mathrm{l})$ were then pipetted onto each grid. After 30 seconds of adsorption time the grid was blotted manually using Whatman 541 filter paper, for approximately $2 \mathrm{~s}$. Blotting time was optimised for each sample. Grids were then plunged into liquid 95 ethane. The frozen grids were stored in liquid nitrogen until required.

The samples were examined using a Gatan 626 cryoholder (Gatan, CA, USA) and Tecnai 12 Transmission Electron Microscope (FEI, Eindhoven, The Netherlands) at an operating 100 voltage of $120 \mathrm{kV}$, with a Mega- view III CCD camera and AnalySIS camera control software (Olympus, Tokyo, Japan) to record the results. At all times low dose procedures were followed, using an electron dose of 8-10 electrons $/ \AA^{-2}$ for all 


\begin{tabular}{|c|c|c|c|c|c|c|}
\hline $\begin{array}{c}\text { DPPS } \\
\text { (wt\%) }\end{array}$ & $0 \%$ & $2 \%$ & $4 \%$ & $8 \%$ & $12 \%$ & $16 \%$ \\
\hline $\begin{array}{c}\text { GMO+ } \\
\text { F127 }\end{array}$ & & & & & & \\
\hline
\end{tabular}

Figure 1 - The influence of 1, 2 - dipalmitoyl phosphatidyl serine (DPPS) on GMO dispersions stabilised with Pluronic ${ }^{\circledR}$ F127.

imaging. Images were recorded using magnifications in the range $30000 \mathrm{X}$ to $97000 \mathrm{X}$.

\subsection{Small-angle $X$-ray scattering}

Small-angle X-ray scattering (SAXS) experiments were 5 performed on the SAXS/WAXS beamline at the Australian Synchrotron. Samples were transferred into individual wells of a 96-well cell culture plate. Samples were exposed to the $12 \mathrm{keV}$ $\mathrm{X}$-ray beam of dimensions $2500 \mu \mathrm{m} \times 130 \mu \mathrm{m}$ and a typical flux of $5 \times 1012$ photons/s and diffraction patterns were recorded 10 using a Pilatus $1 \mathrm{M}$ detector (Dectris, Switzerland). A silver behenate standard was used to calibrate the reciprocal space vector for analysis. Temperature ramping was achieved through a Huber Ministat 230 (Huber, Offenburg, Germany). Data reduction (calibration and integration) was performed using 15 AXcess, a custom-written SAXS analysis program written by Dr. Andrew Heron from Imperial College, London. ${ }^{13}$

\subsection{Cytotoxicity}

For cytotoxicity determinations, vesicle samples were sterilized 20 by passing the nanoparticles through a $0.22 \mu \mathrm{m}$ Millex syringedriven filter unit (Merck Millipore, Cork, Ireland). Due to their size, this was not possible for the GMO-only cubosomes. Hence, these cubosomes were transferred directly to a sterile container immediately following sonication and were handled aseptically 25 from then on.

Human embryonic kidney (Hek) 293 cells were seeded at 20,000 cells per well in 96-well tissue culture plates ( 5 replicates per sample). The lipid formulations at concentrations varying from 0 to $200 \mu \mathrm{g} \mathrm{ml}^{-1}$ were added to the 96 well culture plates 30 and incubated for $48 \mathrm{~h}$ at $37{ }^{\circ} \mathrm{C}$ with $5 \% \mathrm{CO}_{2} / 95 \% \mathrm{O}_{2}$ in $100 \mu \mathrm{l}$ RPMI containing 5\% Fetal Bovine Serum (Gibco, USA), $1 \%$ non-essential amino acids 100x (Gibco, USA), 1\% Glutamax 100x (Gibco, USA) and 1\% Anti-Anti 100x (Gibco, USA). Toxicity was measured using the Alamar Blue reagent 35 (Invitrogen USA). Following the addition of $10 \mu \mathrm{l}$ of Alamar Blue, the cells were incubated for $4 \mathrm{~h}$ at $37{ }^{\circ} \mathrm{C}$ with $5 \% \mathrm{CO}_{2} /$ $95 \% \mathrm{O}_{2}$. The assay was read on a Cary ${ }^{\circledR} 50 \mathrm{UV}-\mathrm{Vis}$ spectrophotometer (Varian, USA) at $570 \mathrm{~nm}$ and $600 \mathrm{~nm}$. Cell viability was determined by subtracting the absorbance at $570 \mathrm{~nm}$ 40 from that at $600 \mathrm{~nm}$. Results are presented as a percentage of untreated cells.

\section{Results \& Discussion}

\subsection{GMO-DPPS stabilisation with F127}

As shown in Figure 1, increasing the DPPS concentration in
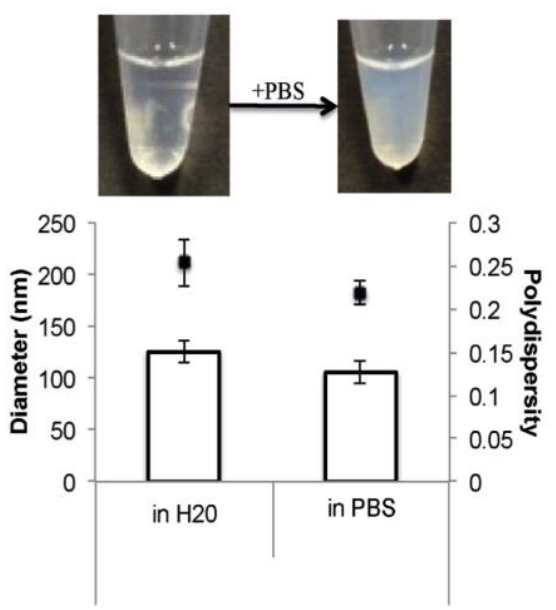

Figure 2 - The addition of PBS to a lamellar GMO-DPPS dispersion stabilised with Pluronic ${ }^{\circledR}$ F127. Top - There is an increase in the optical density of the dispersion following the addition of PBS. Bottom - Diameter (bars) and polydispersity

(points) data obtained through dynamic light scattering displaying a statistically significant change $(\mathrm{p}<0.01)$ in both values.

45 dispersed GMO-F127 mixtures resulted in an optical transition from a milky appearance (typical of cubosomes), to a more translucent/transparent appearance, typical of SUVs. The optical transition occurs when the DPPS concentration reaches $4 \mathrm{wt} \%$, which is in contrast to our previous studies using DPPS with 50 phytantriol, where the concentration of DPPS required to disrupt the cubic phase was $>8 \mathrm{wt} \%{ }^{6}$

The lower concentration of DPPS required to disrupt the GMO cubic phase may suggest that, compared to phytantriol, the GMO cubic phase requires less perturbation in order to transition to the 55 lamellar phase. This hypothesis is supported by the more swollen structure of the pure GMO Pn3m phase, with a lattice parameter of $9.3 \mathrm{~nm},{ }^{14}$ compared to the phytantriol $\mathrm{Pn} 3 \mathrm{~m}$ phase, which has a lattice parameter of $6.79 \mathrm{~nm} .^{14}$

We also note that the stabiliser F127 behaves differently in ${ }_{60}$ GMO dispersions compared to phytantriol dispersions. In GMO dispersions, F127 interacts with and decreases the CPP of the cubic bilayer towards the CPP of the lamellar phase, while in phytantriol dispersions the effect of F127 on the phase behaviour of the system is negligible. This further suggests that F127 65 stabilised GMO dispersions are inherently more susceptible to the DPPS-induced cubic-lamellar phase transition than those of phytantriol.

The addition of PBS to the GMO-4\% DPPS-F127 dispersion results in only a slight change in the optical characteristics of the 70 dispersion as well as a reduction in the diameter and polydispersity of the particles (Figure 2). The typical milky appearance of a cubosome dispersion, which is not observed in this case, would seem to be either the result of an incomplete lamellar - cubic phase transition (i.e. incomplete SUV-to75 cubosome conversion), or the production of smaller, less scattering cubosome particles. This latter feature was previously observed when phytantriol-DPPS cubosomes were produced using the 'salt induced' cubosome production technique. ${ }^{6}$ 


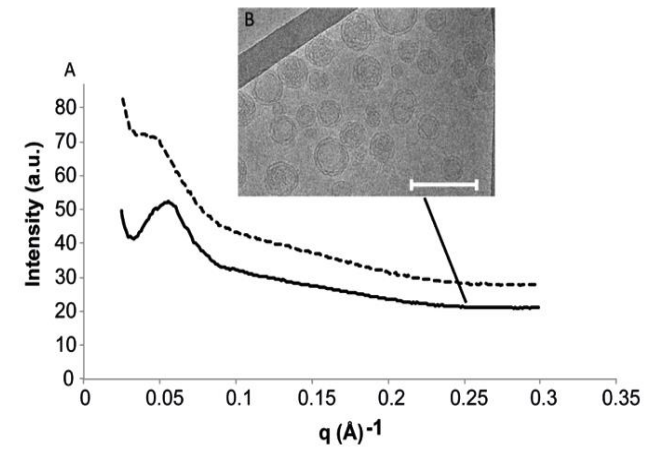

Figure 3 - A - Synchrotron small-angle X-ray scattering (SAXS) of a lamellar GMO-DPPS-F127 dispersion in $\mathrm{H} 2 \mathrm{O}$ (dashed) and the dispersion following the addition of PBS (solid). B- Cryo-TEM image of the GMO-DPPS-F127 dispersion following the addition of PBS shows the dispersion is in a cubic-lamellar phase. Scale bar = $200 \mathrm{~nm}$.

\begin{tabular}{|c|c|c|c|c|}
\hline $\begin{array}{c}\text { DPPS } \\
\text { (wt\%) }\end{array}$ & $\mathbf{0 \%}$ & $\mathbf{2 \%}$ & $\mathbf{4 \%}$ & $\mathbf{8 \%}$ \\
\hline $\begin{array}{c}\mathrm{GMO}+ \\
\mathrm{F} 127\end{array}$ & & & & \\
\hline $\begin{array}{c}\mathrm{GMO}+ \\
\mathrm{F} 108\end{array}$ & & & & \\
\hline
\end{tabular}

Figure 4 - The influence of 1,2 - dipalmitoyl phosphatidyl serine (DPPS) on GMO dispersions stabilised with Pluronic ${ }^{\circledR}$ F127 and Pluronic ${ }^{\circledR}$ F108.

Cryo-TEM and SAXS were employed to characterize the GMO-4\% DPPS-F127 dispersion after the addition of PBS (Figure 3). Both before and after the addition of PBS, the SAXS scatterplot displayed no Bragg peaks, which is as expected for a 5 dispersion of SUVs (Figure 3A). Using cryo-TEM, we observe that following the addition of PBS, the dispersion possesses a mixed cubic - lamellar character (Figure 3B). The high concentration of lamellar phase in the cryo-TEM likely explains why there are no Bragg peaks observable in the SAXS scatterplot 10 and confirms that the small change in optical density is a product of only a partial lamellar-to-cubic phase transition occurring.

We have demonstrated that the addition of a physiologically relevant concentration of PBS does not induce a SUV-tocubosome transition in GMO-4\% DPPS-F127 vesicles. This is

15 likely to be due, at least in part, to the non-electrostatic amphiphile and/or Pluronic ${ }^{\circledR}$ F127 interactions stabilising the lamellar phase system.

It has been shown previously that Pluronic ${ }^{\circledR}$ F108 has limited influence on the GMO cubic phase structure relative to F127. ${ }^{12}$

${ }_{20}$ We therefore hypothesised that employing F108 may eliminate the possible destabilising role of F127, and allow the salt induced lamellar - to - cubic phase transition to occur.

\subsection{GMO-DPPS stabilisation with F108}

${ }_{25}$ Pluronic $^{\circledR}$ F108 is a non-ionic surfactant composed of 50 hydrophobic propylene oxide (PO) units and 132 hydrophilic ethylene oxide (EO) units, making it a slightly larger and more hydrophilic surfactant than F127 (65:100) (Table 1). ${ }^{15}$

The addition of DPPS to GMO-F108 dispersions results in 30 similar changes to those observed in the F127 system (Figure 4).

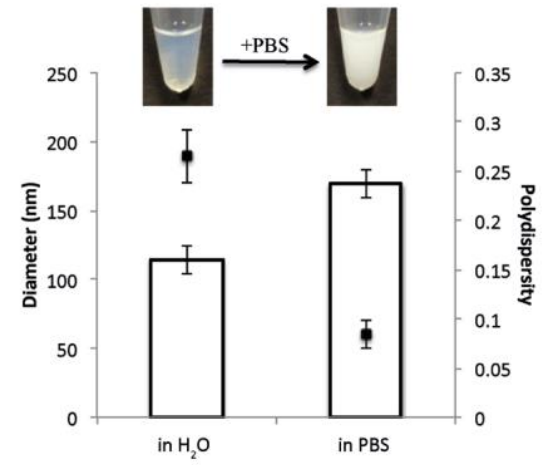

Figure 5 - The addition of PBS to a lamellar GMO-DPPS dispersion stabilised with Pluronic ${ }^{\circledR}$ F108. Top - There is an increase in the optical density of the dispersion following the addition of PBS. Bottom - Diameter (bars) and polydispersity (points) data obtained through dynamic light scattering displaying

a statistically significant change $(p<0.01)$ in both values.

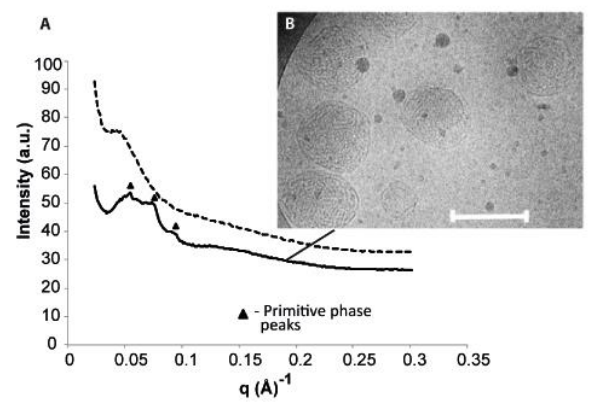

Figure 6 - A - Synchrotron small-angle X-ray scattering (SAXS) of a lamellar GMO-DPPS-F108 dispersion (dashed) and the dispersion following the addition of PBS (solid). B - Cryo-TEM image of the GMO-DPPS-F108 dispersion following the addition of PBS. Scale bar $=200 \mathrm{~nm}$.

When the concentration of DPPS reaches $4 \mathrm{wt} \%$, the dispersion appears translucent and, therefore, presumably comprises SUVs. However, unlike the GMO-4\% DPPS-F127 dispersions, the addition of PBS to the GMO-4\% DPPS-F108 dispersion results in 35 a visible optical transition from the translucent SUV phase to the 'milky' appearance that we associate with cubosomes (Figure 5).

In addition to the visible transition, size analysis using dynamic light scattering demonstrates that the addition of PBS results in significantly larger particles being formed. (Figure 5B). ${ }_{40}$ The size changes we observe here agree with previous work reporting SUV-to-cubic phase transitions, wherein, following the addition of PBS, the diameter of the particles increases and the polydispersity decreases. ${ }^{6,7}$ These changes are caused by the clustering of small unstable cubic phase nanoparticles which form 45 following the addition of PBS. ${ }^{16,17}$

Synchrotron SAXS and cryo-TEM were performed on the dispersions in order to confirm the nature of the transition (Figure 6). Following the addition of PBS, the SAXS scatterplot shows the appearance of low intensity Bragg peaks associated 50 with the primitive $(\mathrm{Im} 3 \mathrm{~m})$ phase and indicates a lattice parameter 

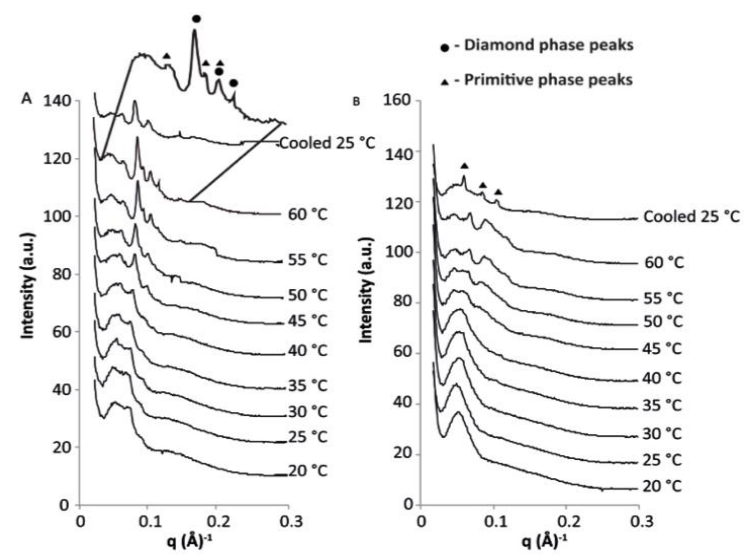

Figure 7 - Phase behaviour of F108 (A) and F127 (B) stabilised GMO-DPPS dispersions at different temperatures as monitored by Synchrotron SAXS. Top: Magnification of GMO-4\% DPPS-

F108 dispersion at $60{ }^{\circ} \mathrm{C}$ displaying primitive and diamond phase peaks.

of $\sim 149 \AA$ (Figure 6A). It is possible that the low Bragg peak intensity is a result of an incomplete/mixed phase transition, as similarly low intensity Bragg peaks have been observed in cubic phase dispersions that contain a large amount of bilayer vesicle ${ }^{5}$ phase. ${ }^{18}$ Although some lamellar phase is still present, cryo-TEM confirms the phase transition from SUVs-to-cubosomes has occurred (Figure 6B). We believe the low-intensity Bragg peaks observed in the SAXS scatterplot above are due to a combination of the low concentration of amphiphile, the small diameter of the 10 cubosomes present in the dispersion and the presence of some lamellar phase.

Nonetheless, the adaptation of the 'salt-induced' cubosome production technique to create cubosomes based on GMO has been demonstrated. This is an important development given that ${ }_{15}$ GMO cubosome formulations have received significant attention in drug delivery applications. This novel production technique may allow the encapsulation of proteins and other biomolecular therapeutics, which are likely to denature under the harsh conditions traditionally used to form cubosomes.

20

\subsection{Temperature stability $20{ }^{\circ} \mathrm{C}-60{ }^{\circ} \mathrm{C}$}

The thermal stability of cubosomes produced through the 'saltinduced' phase transition is an important consideration if they are to be effectively used as drug delivery vehicles. Following 25 injection into the body, drug delivery vehicles are exposed to a range of external factors, which may induce structural change: salts, proteins and temperature to name a few. In the method of cubosome production described above we have attempted to emulate the salt concentration of the human body by using an 30 appropriate concentration of PBS. This is important because changes in the ionic strength will affect the level of charge shielding on the DPPS lipid and, thus, the phase and lattice parameter of the cubosomes. We now discuss the effect of temperature on the stability of GMO-based dispersions produced 35 by the addition of PBS.

In the synchrotron SAXS scatterplot shown in Figure 7A, we

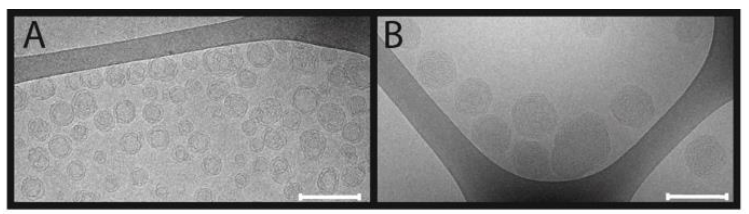

Figure 8 - Cryo-TEM of GMO-F127-DPPS dispersion before (A) and following (B) heat treatment to $60{ }^{\circ} \mathrm{C}$. Scale bar $=200 \mathrm{~nm}$.

observe that, as the temperature of the F108 stabilised GMODPPS dispersion is increased, the Bragg peaks of the cubic phase shift to the right of the scatterplots and eventually exhibit a 40 transition to a diamond-primitive cubic mixed phase, which is most evident at $60{ }^{\circ} \mathrm{C}$ (Figure 7A). Both phenomena correspond to an increase in the CPP of the amphiphiles and a concomitant decrease in water channel diameter with temperature, a common characteristic of cubic phase behaviour. ${ }^{19,20}$

45 Importantly, the SAXS profile of the F108 sample demonstrates that the cubosomes are thermally stable up to 45 $50{ }^{\circ} \mathrm{C}$, which is important if these cubosomes are to be used at 37 ${ }^{\circ} \mathrm{C}$ in the human body.

Interestingly, as the temperature increases over $50{ }^{\circ} \mathrm{C}$, we 50 observe an increase in Bragg peak intensity. This change is evident in both the F108 and F127 stabilised samples (Figure 7A and $\mathbf{B}$, respectively) and the change is similar to that observed in our previous work, where the cooling of phytantriol-DPPS cubosomes lead to an increase in the size of the cubosomes in the ${ }_{55}$ dispersion. $^{6}$

In the phytantriol-DPPS work the increase in size of the cubosomes was caused by phase separation of the Pluronic ${ }^{\circledR}$ stabiliser. There are two critical temperature points for the solubilisation of surfactants. These are the CMT, the temperature ${ }_{60}$ below which the surfactant will form micelles, ${ }^{21}$ and the cloud point, which is the temperature at which the surfactant loses its solubility. ${ }^{22}$ It has been shown previously that cloud point induced phase separation of stabilisers results in increases of cubosome size, however, the temperature required in that work 65 was above $100{ }^{\circ} \mathrm{C} .{ }^{23}$

Although the cloud points of $\mathrm{F} 127$ and $\mathrm{F} 108$ in $\mathrm{H}_{2} \mathrm{O}$ are above $100{ }^{\circ} \mathrm{C},{ }^{22,24}$ it has been demonstrated that by changing the environmental conditions of surfactants, through the addition of salts, the cloud point temperature will decrease. ${ }^{22}$ If this 70 phenomenon has occurred here, we may expect the structure of the cubosomes to change at temperatures below $100{ }^{\circ} \mathrm{C}$. However, the change in Bragg peak intensities in the F127 stabilised dispersion (Figure 7B) suggests that something more than the phase separation of F127 may be occurring.

75 At room temperature the SAXS scatterplot of the GMO-4\% DPPS-F127 dispersion displays no Bragg peaks (Figure 7B). As observed earlier, the GMO-4\% DPPS-F127 dispersion has a large amount of lamellar-bilayer phase present at room temperature (Figure 8A), which gives rise to the scatterplot with no Bragg 80 peaks.

As we heat the dispersion to $45-50{ }^{\circ} \mathrm{C}$ we observe the appearance of Bragg peaks. An increase of temperature naturally increases the CPP of lyotropic liquid crystal bilayers, ${ }^{25}$ and we 
therefore expect to see the CPP of the GMO-4\% DPPS-F127 dispersion driven towards the cubic phase and the appearance of Bragg peaks in the SAXS scatterplot. However, this process should be reversible and, following the cooling process, the 5 Bragg peaks in the scatterplot should disappear, which is not the case in Figure 7B.

To investigate if this change was caused by a slow transition back to the cubic-bilayer phase, cryo-TEM was performed on a GMO-4\% DPPS-F127 dispersion that was heated to $60{ }^{\circ} \mathrm{C}$ for 2 10 hours and then allowed to cool overnight at room temperature (Figure 8B). The cryo-TEM image demonstrates that following heat treatment the particles are larger and have a greater percentage of cubic phase present compared to the untreated sample.

15 These results suggest irreversible changes are occurring in the GMO-4\% DPPS-F127 dispersion following heat treatment, which could be a result of phase separation of F127, phase separation of DPPS or a combination of both.

DPPS has the greatest influence on the CPP of the GMO 20 bilayer and its removal will drive the GMO-4\% DPPS-F127 dispersion towards the cubic phase, which is what we have observed in the scatterplots above. The phase separation of DPPS occurs at $\sim 53{ }^{\circ} \mathrm{C},{ }^{26}$ the gel to liquid crystalline phase change temperature of DPPS, and this is the temperature at which we 25 begin to observe the increase in Bragg peak intensity in both the F108 and F127 samples.

The phase separation of DPPS from the cubic-bilayer particles explains the strong, irreversible cubic phase transition and may also explain the increase in particle size, which we observe in the 30 cryo-TEM images. As discussed above, when cubosomes form following an SUV-to-cubic phase transition we expect to see a clustering of the smaller particles to form stable cubosomes. ${ }^{16} \mathrm{By}$ removing the DPPS through phase separation, we are inducing the SUV-to-cubic phase transition and, therefore, expect this 35 clustering to occur.

The phase separation of DPPS in the GMO-based dispersions reveals that these dispersions do not have the same thermal stability as GMO-only cubosomes. This is an important consideration in the production and storage of cubosomes using 40 the 'salt-induced' production technique as unwanted transitions may occur. However, since the phenomenon arises at temperatures far greater than $37^{\circ} \mathrm{C}$, should these nanoparticles be used for drug delivery purposes, we do not anticipate these issues to be significant in vivo.

\section{${ }_{45}$ 3.4. Cytotoxicity}

It has previously been shown that the addition of DPPS to phytantriol-based dispersions increases their in vitro toxicity. ${ }^{18}$ The authors in this study found that phytantriol dispersions incorporating DPPS displayed a greater toxicity than those 50 without DPPS and hypothesized that this may be due to these dispersions being in a mixed cubic-bilayer phase, similar to the GMO-F127-DPPS nanoparticles described here.

It is therefore important we characterise the toxicity of cubosomes formed using DPPS to confirm that the presence of 55 the anionic lipid does not increase the toxicity of GMO. Also, the toxicity of F108 as a cubosome stabiliser, to our knowledge, remains untested. This surfactant was only recently recognised as having properties suitable for the stabilisation of GMO

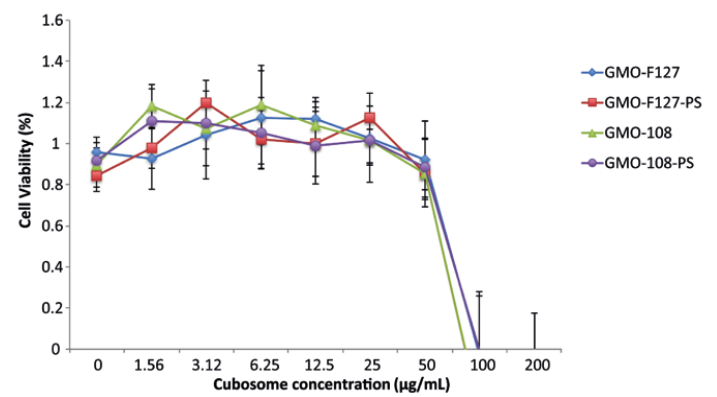

Figure 9 - In vitro cell viability of GMO and GMO-DPPS dispersions stabilised with either Pluronic ${ }^{\circledR}$ F127 or Pluronic ${ }^{\circledR}$ F108. Amphiphile nanoparticles were added to a Hek293 cell line. Error bars $=$ Standard deviation; $\mathrm{n}=5$.

dispersions.

60 Figure 9 demonstrates the cell viability of a Hek293 cell line following the addition of GMO-based cubosomes. We observe there is no loss of cell viability in the Hek 293 cell line up to 50 $\mu \mathrm{g} / \mathrm{ml}$ of cubosomes. This value is in line with other studies that have analysed the toxicity of GMO-based cubosomes with the 65 Hek 293 cell line. ${ }^{27}$

The noticeable difference between this and previous work is the insignificant difference that DPPS has on cell viability. Shen et al. (2010) had previously hypothesised that the increased toxicity, which phytantriol-DPPS nanoparticles exhibit in vitro, 70 may be due to the mixed cubic-lamellar states of the dispersion. This hypothesis was formulated based on the characterization of water-based dispersions before we had elucidated the critical influence of salt concentration on the lamellar-to-cubic phase transition in these systems. As such, this hypothesis is now less 75 compelling, since given the cell viability assay is conducted in a salt based solution, these water-based particles are expected to undergo a phase transition to the cubic phase. There are, however, a range of other factors that influence cell viability assays, which may have lead to the discrepancy in results.

80 It is important to note that the in vitro cytotoxicity of cubosomes may still be a concern at $50 \mu \mathrm{g} / \mathrm{mL}$. The IC50 value, the concentration required to reduce the cell viability by $50 \%$, for other amphiphilic particles can be as high as $2.9 \mathrm{mg} / \mathrm{mL},{ }^{28}$ significantly higher than what we have observed here. In vivo 85 toxicity and delivery studies will therefore be required to further optimise and assess the applicability of these particles for therapeutic delivery applications.

\section{Conclusion}

The preparation of GMO-based cubosomes using the 'salt${ }_{90}$ induced' production technique is an important step in the low energy manufacture of cubosomes for drug delivery applications. The most commonly used stabiliser, Pluronic ${ }^{\circledR}$ F127, was shown to inhibit the required vesicle-to-cubic phase transition of the GMO-DPPS system. However, use of Pluronic ${ }^{\circledR}$ F108, instead of ${ }_{95}$ Pluronic $^{\circledR}$ F127 as a stabiliser for cubosome dispersions restored the 'salt induced' lamellar-to-cubic phase transition, and allowed cubosome formation at a physiologically relevant salt concentration.

Using SAXS analysis we also demonstrated that these 100 cubosomes are thermally stable up to $45^{\circ} \mathrm{C}$, however, above this temperature we believe the phase separation and expulsion of 
DPPS from the particles is causing irreversible changes to the structure of the particles.

In addition, we have shown that the in vitro cytotoxicity of these GMO-based cubosomes, produced using the 'salt-induced' 5 production technique, display no difference in cell viability compared to GMO-only cubosomes, contrary to previous reports using phytantriol and DPPS.

\section{Acknowledgments}

10 T.E. Hartnett would like to thank The University of Melbourne, the Commonwealth Scientific and Industrial Research Organisation (CSIRO), and the Melbourne Materials Institute (MMI) for various scholarships. K. Ladewig acknowledges financial support from the Australian Research Council (ARC) in 15 the form of an ARC Super Science Fellowship (FS 110200025). Infrastructure support from the Particulate Fluids Processing Centre (PFPC), the MMI, CSIRO and The University of Melbourne is gratefully acknowledged. This research was undertaken on the SAXS/WAXS beamline at the Australian 20 Synchrotron, Victoria, Australia (M5720). Cryo-TEM images were obtained with the assistance of Ms. Lynne Waddington, CSIRO, Parkville. Financial support was also provided by the National Health and Medical Research Council (NHMRC, Program Grant 1047603).

25

\section{References}

1. X. Mulet, B. J. Boyd, and C. J. Drummond, J. Colloid Interface Sci., 2013, 393, 1-20.

2. M. Lawrence, Chem. Soc. Rev., 1994, 23, 417-424.

$303 . \quad$ J. C. Shah, Y. Sadhale, and D. M. Chilukuri, Adv. Drug Deliv. Rev., 2001, 47, 229-50.

$4 . \quad$ Q. Liu, Y.-D. Dong, T. L. Hanley, and B. J. Boyd, Langmuir, 2013, 29, 14265-73.

5. F. Muller, A. Salonen, and O. Glatter, J. Colloid Interface Sci., $35 \quad 2010,342,392-8$.

6. T. Hartnett, K. Ladewig, P. G. Hartley, A. J. O'Connor, and K. M. McLean, J. Phys. Chem. B, 2014, 118, 7430-7439.

7. B. W. B. Muir, G. Zhen, P. Gunatillake, and P. G. Hartley, J. Phys. Chem. B, 2012, 116, 3551-3556.

40 8. E. Wagner, Parfum. und Kosmet., 1994, 75, 260-267.

9. H. Qiu and M. Caffrey, Biomaterials, 2000, 21, 223-34.

10. J. Barauskas and T. Landh, Langmuir, 2003, 19, 9562-9565.

11. T. M. Hinton, F. Grusche, D. Acharya, R. Shukla, V. Bansal, L. J. Waddington, P. Monaghan, and B. W. Muir, Toxicol. Res.
12. J. Y. T. Chong, X. Mulet, L. J. Waddington, B. J. Boyd, and C. J. Drummond, Soft Matter, 2011, 7, 4768 - 4777.

13. J. M. Seddon, A. M. Squires, C. E. Conn, O. Ces, A. J. Heron, X. Mulet, G. C. Shearman, and R. H. Templer, Philos. Trans. A. Math. Phys. Eng. Sci., 2006, 364, 2635-55.

14. P. Wadsten-Hindrichsen, J. Bender, J. Unga, and S. Engström, J. Colloid Interface Sci., 2007, 315, 701-13.

15. 2014

16. B. Angelov, A. Angelova, V. M. Garamus, M. Drechsler, R. 55 Willumeit, R. Mutafchieva, P. Štěpánek, and S. Lesieur, Langmuir, 2012, 28, 16647-55.

17. B. Angelov, A. Angelova, B. Papahadjopoulos-Sternberg, S. Lesieur, J.-F. Sadoc, M. Ollivon, and P. Couvreur, J. Am. Chem. Soc., 2006, 128, 5813-7.

$6018 . \quad$ H. Shen, J. Crowston, F. Huber, S. Saubern, K. M. Mclean, and P. G. Hartley, Biomaterials, 2010, 31, 9473-9481.

19. G. Wörle, M. Drechsler, M. H. J. Koch, B. Siekmann, K. Westesen, and H. Bunjes, Int. J. Pharm., 2007, 329, 150-7.

20. J. Zhai, L. Waddington, T. Wooster, M. Aguilar, and B. Boyd, 65 Langmuir, 2011, 27, 14757-14766.

21. M. Bohorquez, C. Koch, T. Trygstad, and N. Pandit, J. Colloid Interface Sci., 1999, 216, 34-40.

22. N. Pandit, T. Trygstad, S. Croy, M. Bohorquez, and C. Koch, J. Colloid Interface Sci., 2000, 222, 213-220.

$7023 . \quad$ J. Barauskas, M. Johnsson, F. Joabsson, and F. Tiberg, Langmuir, 2005, 21, 2569-77.

24. K. Flodström and V. Alfredsson, Microporous Mesoporous Mater., 2003, 59, 167-176.

25. A. Angelova, M. Ollivon, A. Campitelli, and C. Bourgaux, $75 \quad$ Langmuir, 2003, 19, 6928-6935.

$26 . \quad$ Z. D. Schultz, I. M. Pazos, F. K. McNeil-Watson, E. N. Lewis, and I. W. Levin, J. Phys. Chem. B, 2009, 113, 9932-41.

27. B. W. Muir, D. P. Acharya, D. F. Kennedy, X. Mulet, R. a Evans, S. M. Pereira, K. L. Wark, B. J. Boyd, T.-H. Nguyen, T. M. Hinton, L. J. Waddington, N. Kirby, D. K. Wright, H. X. Wang, G. F. Egan, and B. a Moffat, Biomaterials, 2012, 33, 2723-33.

28. E. Mayhew, M. Itos, and R. Lazo, Exp. Cell Res., 1987, 171, 195-202.

Notes and references 
${ }^{a}$ Department of Chemical and Biomolecular Engineering and Particulate Fluids Processing Centre (PFPC), The University of Melbourne,

Parkville, VIC 3010, Australia

${ }^{b}$ Commonwealth Scientific and Industrial Research Organisation 5 (CSIRO), Materials Science and Engineering, Clayton, VIC 3168, Australia

* corresponding author: Keith McLean

Commonwealth Scientific and Industrial Research Organisation (CSIRO),

10 Materials Science and Engineering, Clayton, VIC 3168, Australia

Email: keith.mclean@csiro.au

Phone: +61395452599 


\section{University Library}

\section{- M M I N E R VA A gateway to Melbourne's research publications}

Minerva Access is the Institutional Repository of The University of Melbourne

Author/s:

Hartnett, TE;Ladewig, K;O'Connor, AJ;Hartley, PG;McLean, KM

Title:

Physicochemical and cytotoxicity analysis of glycerol monoolein-based nanoparticles

Date:

2015-01-01

Citation:

Hartnett, T. E., Ladewig, K., O'Connor, A. J., Hartley, P. G. \& McLean, K. M. (2015).

Physicochemical and cytotoxicity analysis of glycerol monoolein-based nanoparticles. RSC ADVANCES, 5 (34), pp.26543-26549. https://doi.org/10.1039/c4ra13890b.

Persistent Link:

http://hdl.handle.net/11343/55187 